\title{
Alterações dimensionais transversas do arco dentário com fissura labiopalatina, no estágio de dentadura decídua*
}

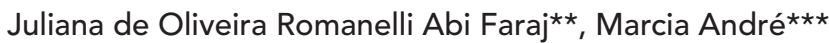

\begin{abstract}
Resumo
Objetivo: avaliar as alterações dimensionais do arco dentário maxilar de crianças portadoras de fissuras labiopalatinas, na fase de dentadura decídua completa, através de um estudo comparativo. Metodologia: foram estudadas as distâncias entre caninos e entre molares em 64 crianças fissuradas, formando o grupo de estudo, e 20 crianças sem deformidades, formando o grupo controle, na faixa etária entre 3 e 6 anos. O grupo de estudo incluiu 23 crianças com fissura transforame incisivo unilateral completa, $14 \mathrm{com}$ fissura transforame incisivo bilateral completa, 15 com fissura pré-forame incisivo e 12 com fissura pós-forame incisivo. Após a obtenção de modelos em gesso, foram demarcados os pontos necessários para que as mensurações pudessem ser realizadas. As medidas foram executadas duas vezes pelo mesmo examinador em oportunidades diferentes, utilizando um paquímetro digital (Mitutoyo), sendo registrada a média aritmética. Os resultados obtidos foram submetidos à análise estatística (Teste F Anova no procedimento Tukey-Kramer). Conclusões: as fissuras transforame incisivo, unilaterais ou bilaterais, por romperem totalmente a maxila, foram as que apresentaram alterações da distância entre caninos, comprometendo significantemente sua dimensão transversal anterior, na fase de dentadura decídua.
\end{abstract}

Palavras-chave: Alterações dimensionais. Arcos dentários. Dentadura decídua. Fissuras labiopalatinas.

\section{INTRODUÇÃO E REVISÃO DE LITERATURA}

As fissuras labiopalatinas são más formações congênitas que acometem o terço médio da face, normalmente comprometendo a estética e boa parte das funções orofaciais, o que implica na necessidade de uma equipe multiprofissional, para que possam ser estabelecidas as bases de uma ampla reabilitação. O conhecimento dos diversos ti- pos de fissura e do comportamento das respectivas estruturas envolvidas é imprescindível para o profissional que se habilita a trabalhar com pacientes fissurados. Estas anomalias são diferenciadas pela extensão da lesão e podem ser classificadas utilizando, como ponto de referência anatômica, o forame incisivo, vestígio embrionário que demarca os limites entre o palato primário e o palato

* Parte da Dissertação de Mestrado apresentada ao curso de Pós-Graduação em Prótese Buco Maxilo Facial da FOUSP.

** Mestre em Prótese Buco Maxilo Facial pela Faculdade de Odontologia da USP. Especialista em Ortodontia e Ortopedia Funcional dos Maxilares, UNICASTELO / SP.

*** Professora Doutora da Disciplina de Prótese Buco Maxilo Facial da FOUSP. Especialidades: Prótese Buco Maxilo Facial e Pacientes com Necessidades Especiais. 
secundário ${ }^{28}$.

As fissuras localizadas à frente do forame incisivo recebem o nome de fissuras pré-forame incisivo e têm origem embriológica do palato primário. Neste grupo incluem-se as fissuras que comprometem o lábio e/ou processo alveolar. São consideradas completas quando envolvem toda a extensão do lábio e rompem o alvéolo, alcançando o assoalho nasal até atingir o forame incisivo; caso contrário, são classificadas como incompletas, podendo ser uni ou bilaterais (Fig. 1).

As fissuras que acometem o palato secundário são chamadas de fissuras pós-forame incisivo, sendo consideradas completas quando acometem o palato duro e mole ou incompletas quando não rompem todo o palato (Fig. 2).

As fissuras transforame incisivo se caracterizam por comprometerem o palato primário e o secundário, de forma completa, uni ou bilateralmente (Fig. 3).

O tratamento de fissurados tem sido um constante desafio, que deve ser iniciado ao nascimento, visando a melhor adequação da cavidade bucal a todas as demandas funcionais. A constatação de diferenças existentes na morfologia dos arcos dentários em indivíduos fissurados é freqüentemente abordada pela literatura e a maior parte dos trabalhos avalia o crescimento maxilar de forma longitudinal 2,10,14,17,18,21,22,29,30.

A alteração do crescimento craniofacial destes indivíduos é relatada como resultado natural da presença da fissura ${ }^{30}$, promovendo mudanças na morfologia dos ossos maxilares e conseqüentemente dos arcos dentários, embora Capelozza Filho, Martins e Brosco ${ }^{6}$ afirmem ser normal o potencial de crescimento maxilar destes indivíduos.

O paciente portador de fissura labiopalatina apresenta alterações no complexo maxilofacial que são atribuídas a uma série de fatores. Além de condições pré-estabelecidas, como a gravidade da fissura ${ }^{6,13,15,16,21,30}$ e os padrões genéticos de crescimento $^{3,6}$, há um conjunto de outros fatores que podem produzir alterações nas dimensões de seus arcos dentários, podendo ou não atuar simultaneamente.

As cirurgias reparadoras primárias (queiloplastia e palatoplastia) têm como objetivo restabelecer a estética facial e permitir o desenvolvimento correto das funções orais: sucção, deglutição, mastigação, fonação e respiração. Estas podem ser favoráveis, desde que não causem tensão tecidual, reposicionando os segmentos maxilares, beneficiando o crescimento e o desenvolvimento das estruturas orofaciais ${ }^{3}$.

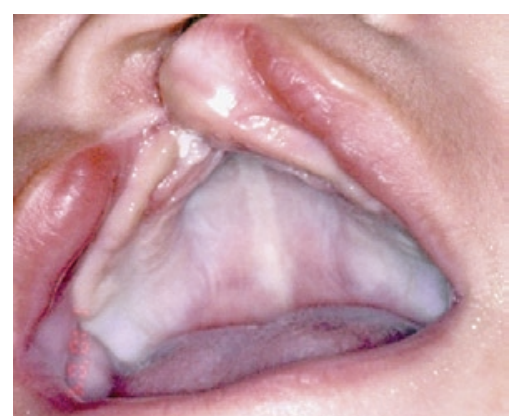

FIGURA 1 - Fissura pré-forame incisivo unilateral completa.

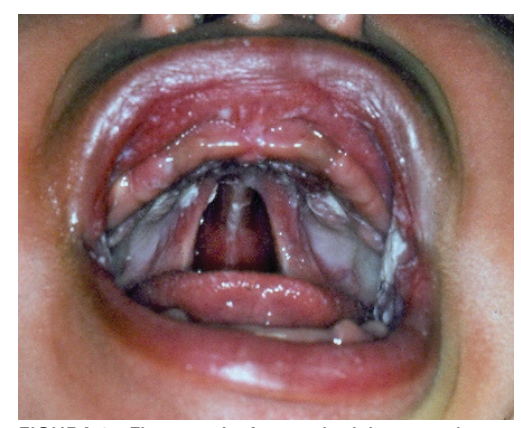

FIGURA 2 - Fissura pós-forame incisivo completa.

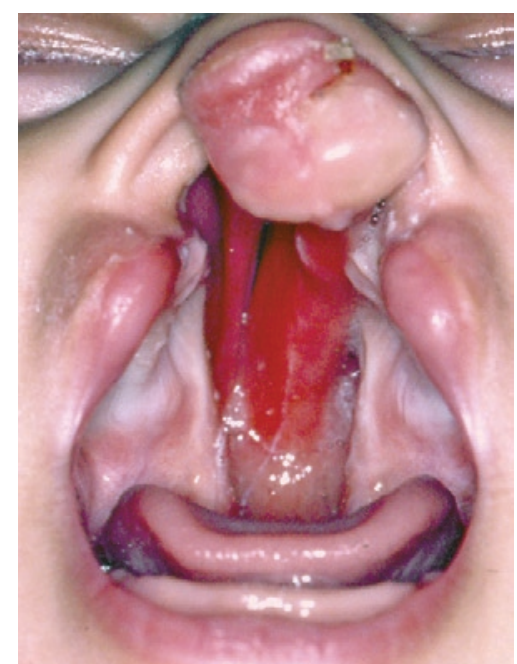

FIGURA 3 - Fissura transforame incisivo bilateral completa. 
É importante salientar que as cirurgias primárias exercem influência de grau variável, na dependência da extensão das fissuras. Desta forma, fissuras isoladas e incompletas de lábio estão pouco sujeitas aos efeitos lesivos dos procedimentos cirúrgicos $^{13}$. Alguns trabalhos ${ }^{15,18}$ mostram que, nem em fissuras isoladas de palato, existe um comportamento restritivo do crescimento maxilar em função da cirurgia reparadora.

Entretanto, nas fissuras transforame incisivo, a maxila, desestabilizada pela sua ruptura, constituindo-se de dois segmentos em fissuras unilaterais e em três nas bilaterais, sofre alterações mais significantes na morfologia dos arcos dentários, decorrentes da terapêutica cirúrgica ${ }^{7,14}$.

A técnica cirúrgica e o número de interven-

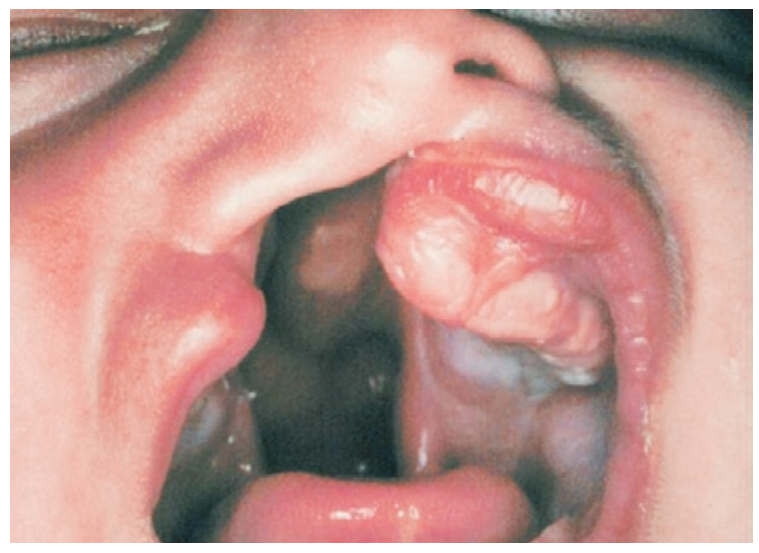

FIGURA 4 - Fissura transforame incisivo unilateral (FLPU).

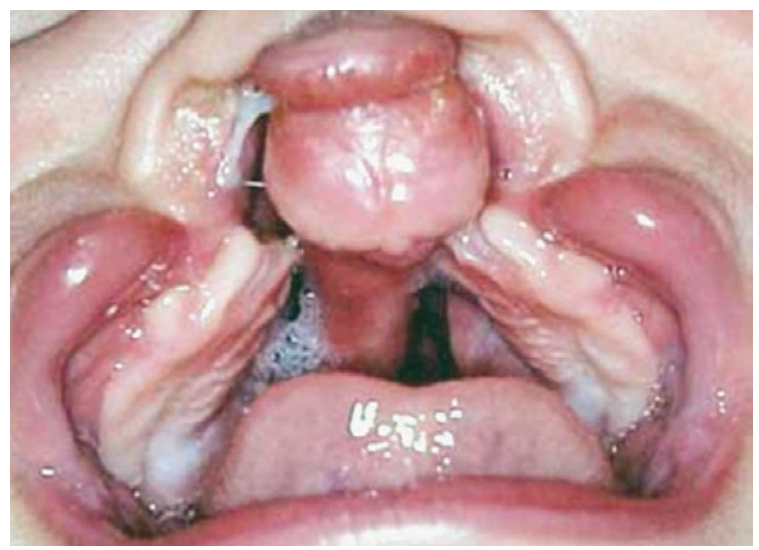

FIGURA 5 - Fissura transforame incisivo bilateral completa (FLPB). ções sofrem influência da gravidade da fissura ${ }^{3,19,21}$, sendo relatados como fatores determinantes na diferenciação das alterações ocorridas nos arcos dentários. A época em que elas devem ser executadas também pode interferir ${ }^{20,21,27,29}$, porém alguns resultados demonstraram que, a longo prazo, não há diferenças consideradas significantes ${ }^{22}$. Além disso, o conhecimento e a habilidade do cirurgião-plástico e até mesmo o ambiente sócio-econômico de cada indivíduo também foram considerados ${ }^{3,6}$.

O fato de o cirurgião-dentista estar ciente das alterações dimensionais ocorridas nos arcos superiores de crianças fissuradas é de extremo interesse terapêutico, tanto no que se refere à Ortodontia e à Odontologia em geral, quanto à interação multiprofissional necessária, pois pode influenciar na
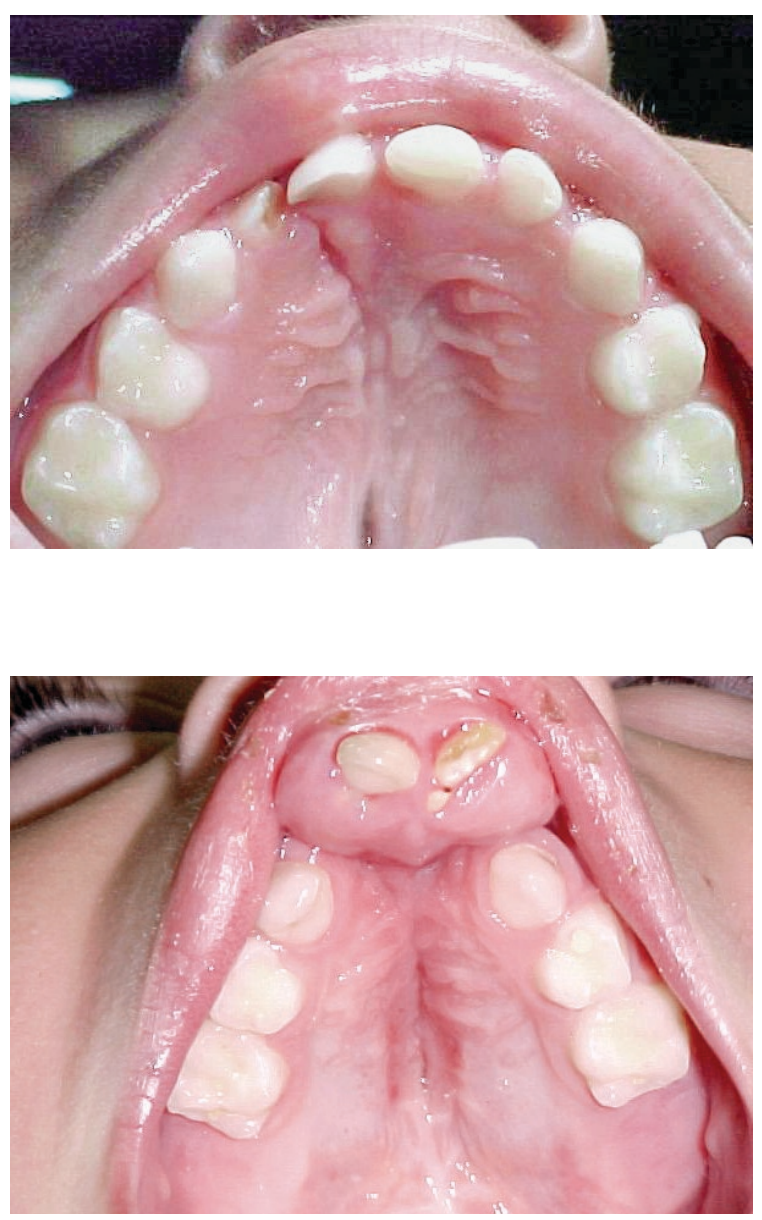
estabilidade dos resultados obtidos na reabilitação global. O presente trabalho teve o intuito de realizar um estudo comparativo das alterações dimensionais do arco dentário superior na fase de dentadura decídua completa, através da mensuração das distâncias entre caninos e entre molares em fissurados e em crianças que não apresentam qualquer deformidade craniofacial.

\section{MATERIAL E MÉTODOS}

A amostra foi constituída de modelos em gesso de arcos dentários superiores de 64 crianças, selecionadas no ambulatório de fissuras da Disciplina de Prótese Buco Maxilo Facial do Departamento de Cirurgia, Prótese e Traumatologia Maxilo Facial da Faculdade de Odontologia da Universidade de

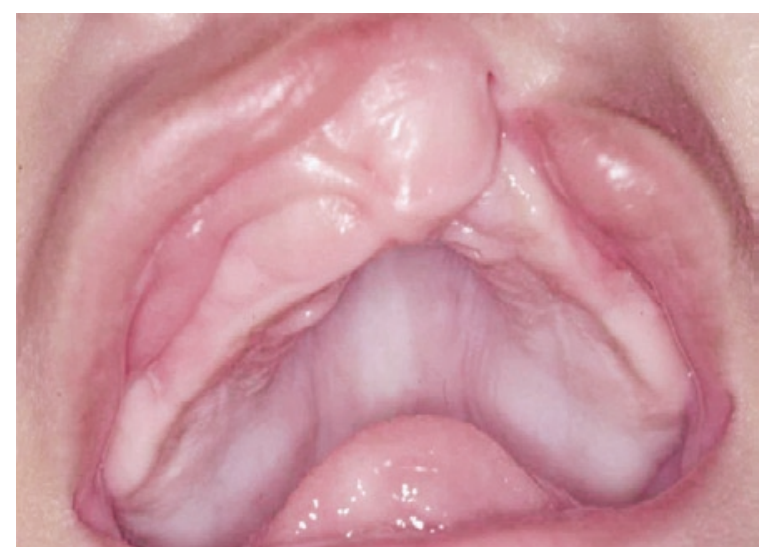

FIGURA 6 - Fissura pré-forame incisivo unilateral completa (FL).

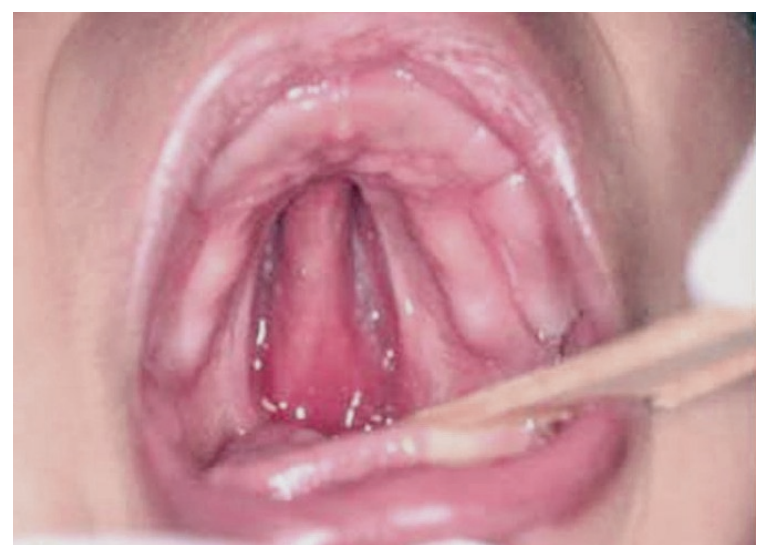

FIGURA 7 - Fissura pós-forame incisivo completa (FP).
São Paulo. Estas crianças eram portadoras de fissuras labiopalatinas já reparadas cirurgicamente, na cronologia clássica (lábio entre 3 e 6 meses, palato entre 12 e 18 meses), em fase de dentadura decídua completa, caracterizando o grupo de estudo. O grupo controle foi formado por 20 crianças sem deformidades craniofaciais e em normoclusão, também com dentadura decídua completa. Em ambos os grupos não houve distinção de gênero ou raça, sendo que a média de idade no grupo de estudo foi de 4 anos e 2 meses e no grupo controle foi de 4 anos e 7 meses. Os fatores de inclusão na pesquisa foram: idade a partir de 3 anos, sem perda de qualquer dente decíduo, por cárie, trauma ou esfoliação, permitindo apenas a ausência dos incisivos laterais adjacentes à fissura, seja por atraso
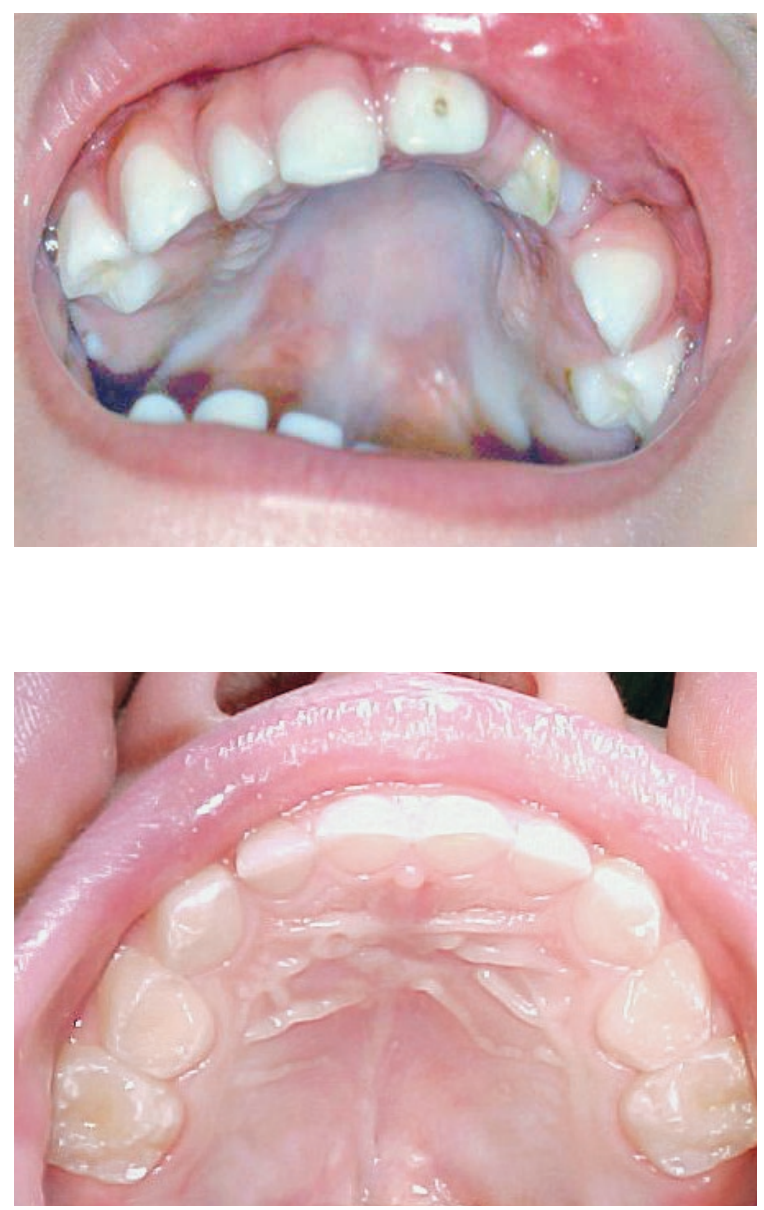
de erupção, seja por agenesia. A presença do primeiro molar permanente, ainda que no início de erupção, constituiu um fator de exclusão, por caracterizar o início da dentadura mista.

As crianças fissuradas foram distribuídas da seguinte forma: 37 com fissuras transforame incisivo, sendo 23 unilaterais (FLPU) (Fig. 4) e 14 bilaterais (FLPB) (Fig. 5); 15 com fissuras pré-forame incisivo completas (FL), todas unilaterais (Fig. 6), e 12 com fissuras pós-forame incisivo completas (FP) (Fig. 7)

Assim que os grupos foram estabelecidos, cada criança teve o arco dentário superior moldado com hidrocolóide irreversível (alginato Jeltrate Plus Dentsply), para obtenção de modelo em gesso pedra Vigodent. As mensurações foram executadas com um paquímetro digital da marca Mitutoyo (precisão de $0,01 \mathrm{~mm})$, duas vezes pelo mesmo operador em oportunidades diferentes, evitando-se assim erros experimentais, sendo registrada a média aritmética dos valores obtidos. A metodologia aplicada baseouse na utilizada por Moorrees ${ }^{23}$, onde a distância entre caninos foi determinada pelo valor em $\mathrm{mm}$ da distância entre os pontos $\mathrm{C}$ e C', localizados nos ápices das cúspides dos caninos decíduos, direito e esquerdo respectivamente, e a distância entre molares pelo valor em mm da distância entre os pontos $\mathrm{M}$ e M', localizados nos ápices das cúspides mesiopalatinas dos segundos molares decíduos, direito e esquerdo respectivamente (Fig. 8).

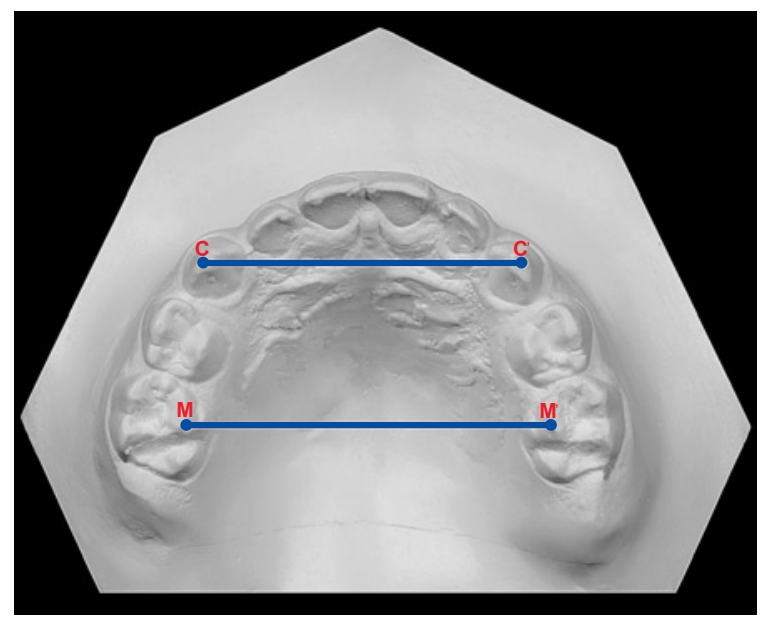

FIGURA 8 - Modelo em gesso do arco superior.
Em algumas crianças, as cúspides dos dentes encontravam-se fisiologicamente desgastadas e, nestes casos, os pontos em estudo foram demarcados nos centros das superfícies abrasionadas dos referidos dentes.

Após serem obtidas as mensurações dos fissurados, suas médias foram registradas e tabeladas de acordo com o tipo de fissura, para que pudessem ser avaliadas e comparadas às do grupo controle. A análise estatística foi efetuada utilizando-se o Teste F Anova com o procedimento Tukey-Kramer.

\section{RESULTADOS}

Os resultados do estudo comparativo da distância entre caninos no arco superior podem ser verificados nas tabelas 1 e 2 . A variabilidade dos valores individuais dessa distância no grupo controle e nos diferentes tipos de fissura pode ser vista no gráfico 1 .

As tabelas 3 e 4 apresentam as distâncias entre molares no arco superior. Por meio do gráfico 2, é possivel conhecer os seus valores individuais em ambos os grupos.

\section{DISCUSSÃO}

As alterações dimensionais ocorridas ao longo do crescimento são consideradas mecanismos compensatórios para manter o equilíbrio entre as diversas demandas funcionais ${ }^{4}$.

A literatura tem demonstrado que a fase de dentadura decídua, na população em geral, não apresenta alterações depois de estabeleci$\mathrm{da}^{9}$ ou, quando apresenta, não são consideradas significantes ${ }^{8,25}$. Por esta razão, acreditou-se que um estudo transversal seria possível, abrangendo a fase de dentadura decídua completa.

A idade cronológica não foi definida como requisito básico da amostra, e sim a presença de todos os dentes decíduos, sem qualquer primeiro molar permanente que pudesse caracterizar o início da dentadura mista, a qual parece ser aceita como a época em que as alterações das dimensões 
Tabela 1 - Média aritmética, desvio-padrão e coeficiente de variação das distâncias entre caninos no arco superior.

\begin{tabular}{cccccc}
\hline Medidas & $\mathbf{C}$ (G Cont.) & $\mathbf{E}_{1}(\mathbf{F L P U})$ & $\mathbf{E}_{2}(\mathbf{F L P B})$ & $\mathbf{E}_{3}(\mathbf{F L})$ & $\mathbf{E}_{4}(\mathbf{F P})$ \\
\hline $\begin{array}{c}\text { média } \\
\text { aritmética }\end{array}$ & 28,861 & 25,939 & 25,719 & 29,802 & 28,284 \\
desvio-padrão & 2,169 & 2,775 & 3,092 & 1,708 & 2,328 \\
$\begin{array}{c}\text { coeficiente de } \\
\text { variação }\end{array}$ & $7,52 \%$ & $10,70 \%$ & $12,02 \%$ & $5,73 \%$ & $8,23 \%$ \\
\hline
\end{tabular}

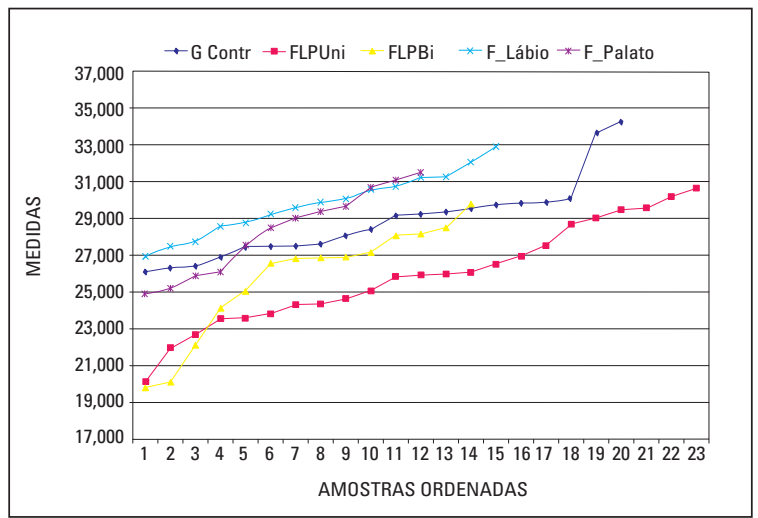

GRÁFICO 1 - Valores em mm das distâncias entre caninos no arco superior de ambos os grupos.

Tabela 2 - Comparação entre os grupos fissurados e controle - número de elementos da amostra, valores (mm) da diferença absoluta e intervalo crítico, resultado da estatística das distâncias entre caninos no arco superior.

\begin{tabular}{|c|c|c|c|c|c|c|c|c|c|c|c|}
\hline $\begin{array}{c}\text { C } \\
(n=20)\end{array}$ & $\mathrm{x}$ & $\begin{array}{c}E_{1} \\
(n=23)\end{array}$ & $\begin{array}{c}\text { C } \\
(n=20)\end{array}$ & $\mathbf{x}$ & $\begin{array}{c}E_{2} \\
(n=14)\end{array}$ & $\begin{array}{c}\text { C } \\
(\mathrm{n}=20)\end{array}$ & $\mathbf{x}$ & $\begin{array}{c}\mathbf{E}_{3} \\
(n=15)\end{array}$ & $\begin{array}{c}\text { C } \\
(n=20)\end{array}$ & $\mathbf{x}$ & $\begin{array}{c}\mathrm{E}_{4} \\
(\mathrm{n}=12)\end{array}$ \\
\hline D.A. & I & I.C. & D.A. & 1 & I.C. & D.A. & I & I.C. & D.A. & I & I.C. \\
\hline 2,921 & 1 & 2,337 & 3,142 & 1 & 2,664 & 0,942 & I & 2,611 & 0,576 & I & 2,792 \\
\hline \multicolumn{3}{|c|}{1,250} & \multicolumn{3}{|c|}{1,179} & \multicolumn{3}{|c|}{0,354} & \multicolumn{3}{|c|}{0,206} \\
\hline \multicolumn{3}{|c|}{ significante } & \multicolumn{3}{|c|}{ significante } & \multicolumn{3}{|c|}{ não significante } & \multicolumn{3}{|c|}{ não significante } \\
\hline
\end{tabular}

Tabela 3 - Média aritmética, desvio-padrão e coeficiente de variação das distâncias entre molares no arco superior.

\begin{tabular}{cccccc}
\hline Medidas & $\begin{array}{c}\mathbf{C} \\
\text { (G Cont.) }\end{array}$ & $\mathbf{E}_{\mathbf{1}}$ (FLPU) & $\mathbf{E}_{\mathbf{2}}$ (FLPB) & $\mathbf{E}_{\mathbf{3}}$ (FL) & $\mathbf{E}_{\mathbf{4}}$ (FP) \\
\hline média aritmética & 33,631 & 32,578 & 33,625 & 34,679 & 34,295 \\
desvio-padrão & 2,157 & 2,899 & 2,004 & 2,134 & 3,069 \\
$\begin{array}{c}\text { coeficiente de } \\
\text { variação }\end{array}$ & $6,41 \%$ & $8,90 \%$ & $5,96 \%$ & $6,15 \%$ & $8,95 \%$ \\
\hline
\end{tabular}

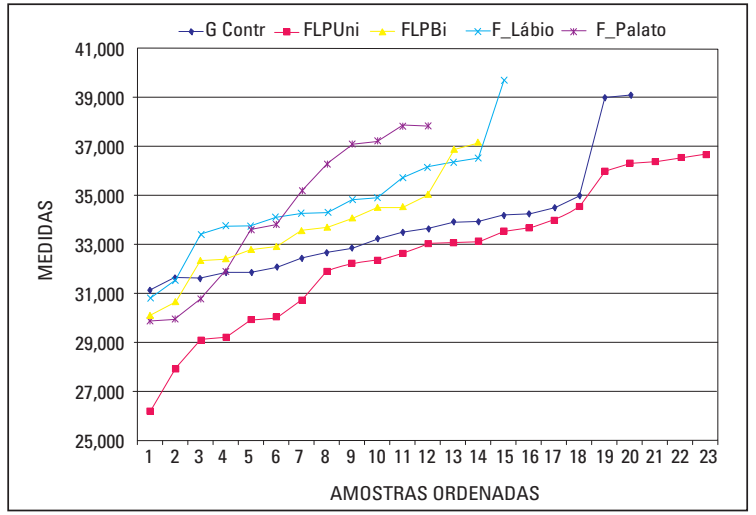

GRÁFICO 2 - Valores em mm das distâncias entre molares no arco superior de ambos os grupos.

Tabela 4 - Comparação entre os grupos fissurados e controle - número de elementos da amostra, valores (mm) da diferença absoluta e intervalo crítico, resultado da estatística para distância entre molares no arco superior.

\begin{tabular}{|c|c|c|c|c|c|c|c|c|c|c|c|}
\hline $\begin{array}{c}\text { C } \\
(\mathrm{n}=20)\end{array}$ & $\mathbf{x}$ & $\begin{array}{c}\mathrm{E}_{1} \\
(\mathrm{n}=23)\end{array}$ & $\begin{array}{c}\text { C } \\
(n=20)\end{array}$ & $\mathbf{x}$ & $\begin{array}{c}E_{2} \\
(n=14)\end{array}$ & $\begin{array}{c}\text { C } \\
(n=20)\end{array}$ & $\mathbf{x}$ & $\begin{array}{c}\mathrm{E}_{3} \\
(\mathrm{n}=15)\end{array}$ & $\begin{array}{c}\text { C } \\
(\mathrm{n}=20)\end{array}$ & $\mathbf{x}$ & $\begin{array}{c}E_{4} \\
(n=12)\end{array}$ \\
\hline D.A. & 1 & I.C. & D.A. & 1 & I.C. & D.A. & 1 & I.C. & D.A. & 1 & I.C. \\
\hline 1,053 & I & 2,36 & 0,006 & I & 2,69 & 1,048 & I & 2,636 & 0,664 & I & 2,818 \\
\hline \multicolumn{3}{|c|}{0,446} & \multicolumn{3}{|c|}{0,002} & \multicolumn{3}{|c|}{0,397} & \multicolumn{3}{|c|}{0,236} \\
\hline \multicolumn{3}{|c|}{ não significante } & \multicolumn{3}{|c|}{ não significante } & \multicolumn{3}{|c|}{ não significante } & \multicolumn{3}{|c|}{ não significante } \\
\hline
\end{tabular}


transversais começam a ocorrer, provavelmente sendo justificadas pela erupção dos primeiros dentes permanentes ${ }^{10,13,24}$.

Avaliando pacientes fissurados, tem sido verificado que cada tipo de fissura apresenta características que resultam em deformidades variadas, únicas ou combinadas, comprometendo os arcos dentários, devido a fatores intrínsecos e extrínsecos à fissura, atuando de forma contínua ou intermitente.

O tipo de fissura é determinante para o desenvolvimento da região anterior da maxila e a redução desta dimensão é diretamente proporcional à gravidade da fissura. Nos casos de fissuras isoladas ou incompletas de palato, existe uma tendência de normalizar o crescimento da largura anterior do arco após os 18 meses de idade. Em relação aos efeitos das cirurgias reparadoras primárias, a palatoplastia não é o procedimento cirúrgico que mais contribui para a redução da largura do arco, porque esta já se apresenta diminuída entre $9 \mathrm{e}$ 12 meses de idade, portanto anteriormente à reparação do palato. Desta forma a queiloplastia é a maior responsável pelas alterações encontradas, uma vez que, em geral, ela é realizada nos primeiros meses de vida ${ }^{21}$.

As cirurgias reparadoras impõem uma certa força restritiva ao crescimento do arco superior ao criar um novo balanço das forças musculares. Este fato ocorre mais devido ao fechamento do lábio do que do palato, alterando mais a distância entre caninos do que a distância entre molares. As mudanças no tamanho e forma do arco superior parecem ocorrer rapidamente, logo após a cirurgia, quando então permanece com seu crescimento relativamente constante até por volta dos cinco anos de idade ${ }^{17}$.

A queiloplastia afeta diretamente a largura anterior do arco, enquanto os efeitos adversos da palatoplastia são de graus variáveis ${ }^{12}$, podendo, quando efetuada tardiamente, favorecer a distância entre caninos ${ }^{20}$.

Como já citado anteriormente, todas as crian- ças desta pesquisa foram submetidas a cirurgias reparadoras na cronologia clássica, porém a corelação entre as alterações dimensionais e o tempo e a técnica cirúrgica não foi o objetivo deste trabalho.

Os resultados observados neste estudo, quanto à distância entre caninos no arco superior, demonstraram que os grupos com fissuras labiopalatinas completas, unilaterais e bilaterais, foram os mais alterados em relação ao grupo controle, apresentando valores menores e estatisticamente significantes. Este fato provavelmente ocorreu devido à perda de continuidade do rebordo alveolar e à ascendência dos segmentos laterais fissurados, características das fissuras completas labiopalatinas, que tendem a interferir na estabilidade da oclusão, tornando-a mais suscetível aos desequilíbrios das forças musculares. Desta forma, os resultados obtidos para as fissuras de palato, que mostraram distâncias entre caninos mais próximas às do grupo controle, podem ser justificados pela integridade do rebordo alveolar.

A distância entre caninos nas fissuras de lábio, apresentaram-se, em média, levemente aumentadas em relação ao grupo controle, o que poderia ser compreendido pelas considerações citadas anteriormente, aliadas à presença freqüente de um dente supranumerário na região da fissura alveolar.

As características da dentadura decídua ou permanente, quanto às dimensões dos arcos, variam conforme a população estudada e a técnica utilizada para realizar as mensurações ${ }^{1}$. Isto é muito mais evidente nas grandezas transversais de indivíduos fissurados, particularmente na largura posterior da maxila, pois alguns trabalhos usam a distância intertuberosidades ${ }^{14,19,20}$, enquanto outros a distância entre molares ${ }^{1,2,5,7,8,9,11,12,18,24,25,26,27}$. Além disso, as diferentes metodologias empregadas para a mensuração da distância entre molares, tendem a dificultar o estudo comparativo.

Nesta pesquisa, foi verificado que as distâncias entre molares do arco superior nas fissuras 
labiopalatinas, em geral, não apresentaram alterações significantes, quando comparadas ao grupo controle.

Independente do tipo de fissura, mordidas cruzadas laterais foram observadas neste estudo, mas raramente com envolvimento dos segundos molares decíduos, o que poderia justificar os resultados das médias das distâncias entre molares da maxila diferirem, em parte, daqueles encontrados na literatura especializada ${ }^{2,11,18,26}$. As técnicas cirúrgicas evoluíram muito ao longo dos anos e os resultados encontrados têm sido mais satisfatórios, em diversos aspectos. $\mathrm{O}$ grupo estudado vem sendo operado em hospitais de referência e por profissionais experientes. Além disso, esta amostra, embora não tivesse sido submetida a algum tipo de tratamento ortopédico, recebia periodicamente orientação odontológica e fonoaudiológica quanto aos aspectos corretos das funções orais, o que, de certa forma, poderia ter favorecido o desenvolvimento maxilar.

\section{CONCLUSÕES}

Frente aos resultados obtidos, na fase de dentadura decídua completa é possível concluir que as fissuras transforame incisivo, uni ou bilaterais, por romperem totalmente a maxila, foram as que apresentaram alterações da distância entre caninos, comprometendo significantemente a sua dimensão transversal anterior.

\title{
Transversal dimensional changes of cleft lip and/or palate arch, in the primary dentition
}

Enviado em: março de 2006 Revisado e aceito: março de 2007

\begin{abstract}
Objective: To evaluate changes in maxillary transversal arch dimension in children with cleft lip and palate, during the complete deciduous dentition period, by a comparative study. Method: This study examined the intercanine and intermolar widths in 64 children with cleft lip and palate, as a study group, and 20 children presenting no deformities as a control group. Children were from 3 to 6 years old. The study group comprised 23 children with unilateral cleft lip and palate (UCLP), 14 with bilateral cleft lip and palate, 15 with cleft lip (CL) and 12 with cleft palate (CP). Dental casts were obtained from both groups and the main points were marked for measurement. Using a digital caliper (Mitutoyo) the same examiner measured each cast twice at different moments and recorded the arithmetic mean. The results were subjected to statistical analysis (Anova $\mathrm{F}$ test within Tukey-Kramer procedure). Conclusions: The UCLP and BCLP presented intercanine width changed, affecting significantly the anterior maxillary transversal dimension during the deciduous dentition period, because they broke the whole maxillary structure.
\end{abstract}

Key words: Dimensional changes. Dental arches. Primary dentition. Cleft lip and palate. 


\section{REFERÊNCIAS}

1. ABU ALHAIJA, E. S.; QUDEIMAT, M. A. Occlusion and tooth/ arch dimensions in the primary dentition of preschool Jordanian children. Int. J. Paediatr. Dent., Oxford, v. 13, no. 14, p. 230-239, July 2003 .

2. ATHANASIOU, A. E.; MAZAHERY, M.; ZARRINIA, K. Longitudinal study of the dental arch dimensions in bilateral cleft lip and palate patients. J. Pedod., Boston, v. 11, p. 253-268, 1987.

3. ATHANASIOU, A. E.; MAZAHERY, M.; ZARRINIA, K. Dental arch dimensions in patients with unilateral cleft lip and palate. Cleft Palate J., Pittsburgh, v. 25, no. 2, p. 139-145, Apr. 1988.

4. BISHARA, S. E.; KHADIVI, P.; JAKOBSEN, J. R. Changes in tooth size: arch length relationships from the deciduous to the permanent dentition: a longitudinal study. Am. J. Orthod. Dentofacial Orthop., St. Louis, v. 108, no. 6, p. 607-613, Dec. 1995

5. BISHARA, S. E.; JAKOBSEN, J. R.; TREDER, J.; NOWAK, A. Arch width changes from 6 weeks to 45 years of age. Am. J. Orthod. Dentofacial Orthop., St. Louis, v. 111, no. 4, p. 401409, 1997

6. CAPELOZZA FILHO, L. MARTINS, D. R.: BROSCO, H. B. Alterações do arco mandibular em portadores de fissura transforame incisivo unilateral. Ortodontia, São Paulo, v. 11, no. 3, p. 160-168, set./dez. 1978

7. DIBIASE, A. T.; DIBIASE, D. D.; HAY, N. J.; SOMMERLAD, B. C. The relationship between arch dimensions and 5-year index in the primary dentition of patients with complete UCLP. Cleft Palate Craniofac., Pittsburgh, v. 39, no. 6, p. 635-640, Nov. 2002

8. DINELLI, T. C. S.; MARTINS, L. P.; PINTO, A. S. Mudanças dimensionais dos arcos dentários em crianças entre 3 e 6 anos de idade. R. Dental Press Ortodon. Ortop. Facial, Maringá, v. 9, no. 4, p. 60-67, jul./ago. 2004.

9. FACAL-GARCÍA, M.; NOVA-GARCÍA, J.; SUÁREZ-QUINTANILLA, D. The diastemas in deciduous dentition: The relationship to the tooth size and the dental arch dimensions. J. Clin. Pediatr. Dent., Birmingham, v. 26, no. 1, p. 65-69, Fall 2001.

10. FOSTER, T. D.; GRUNDY, M. C.; LAVELLE, C. L. B. A longitudinal study of dental arch growth. Am. J. Orthod., St. Louis, v. 72 , no. 3, p. 309-314, Sept. 1977

11. FRIEDE, H.; PERSON, E. C.; LILJA, J.; ELANDER, A.; LOHMANDER-AGERKOV, A.; SÖDERPALM, E. Maxillary dental arch and occlusion in patients with repaired clefts of the secondary palate. Scand. J. Plast. Reconstr. Surg., Stockholm, v. 27, no. 4, p. 297-305, Dec. 1993

12. GARRAHY, A.; MILLETT, D. T.; AYOUB, A. F. Early assessment of dental arch development in repaired unilateral cleft lip and unilateral cleft lip and palate versus controls. Cleft Palate Craniofac. J., Pittsburgh, v. 42, no. 4, p. 385-391, July 2005

13. GOMIDE, M. R.; ABDO, R. C. C. Análise do crescimento do arco maxilar superior em portadores de fissura pré-forame incisivo incompleta. Estudo longitudinal (3 meses -6 anos). Rev. Fac. Odont. Univ. S. Paulo, Bauru, v. 4, no. 1/2, p. 4145, jan. 1996.

14. HEIDBUCHEL, K. L. W. M.; KUIJPERS-JAGTMAN, A. M. KRAMER, G. J. C.; PRAHL-ANDERSEN, B. Maxillary arch dimensions in bilateral cleft lip and palate from birth until four years of age in boys. Cleft Palate Craniofac. J., Pittsburgh, v. 35, no. 3, p. 233-239, May 1998.

15. HELIÖVAARA, A.; RAUTIO, J. Dental arches in six-year-old children with operated and unoperated submucous cleft palate and isolated cleft palate. Acta Odontol. Scand., Oslo, v. 63, p. $123-126,2005$

16. HELLQUIST, R.; PONTÉN, B.; SKOOG, T. The influence of cleft length and palatoplasty on the dental arch and the deciduous occlusion in cases of clefts of the secondary palate. Scand. J. Plast. Reconstr. Surg., Stockholm, v. 12, no. 1, p. 45-54, 1978.
17. HUDDART, A. G.; HUDDART, A. M. An investigation to relate the overall size of the maxillary arch and the area of palatal mucosa in cleft lip and palate cases at birth to the overall size of the upper dental arch at five years of age. J. Craniofac. Genet. Dev. Biol., Copenhagen, v. 1, p. 89-95, 1985. Supplement 1.

18. JONSSON, G.; THILANDER, B. Occlusion, arch dimensions, and craniofacial morphology after palatal surgery in a group of children with cleft in a secondary palate. Am. J. Orthod. St. Louis, v. 76, no. 3, p. 243-255, Sept. 1979.

19. JONSSON, G.: STENSTRÖM S.; THILANDER, B. The use of a vomer flap covered with an autogenous skin graft as a part of the palatal repair in children with unilateral cleft lip and palate. Scand. J. Plast. Reconstr. Surg., Stockholm, v. 14, no. 1, p. 13-21, 1980

20. JORGERSON, R. J.; SHAPIRO, S. D.; ODINET, K. L. Studies on facial growth and arch size in cleft lip and palate. J. Craniofac. Genet. Dev. Biol., Copenhagen, v. 4, no. 1, p. 33-38, 1984.

21. KRAMER, G. J. C.; HOEKSMA, J. B.; PRAHL-ANDERSEN, B. Early palatal changes after initial palatal surgery in children with cleft lip and palate. Cleft Palate Craniofac. J., Pittsburgh, v. 33, no. 2, p. 104-111, Mar. 1996.

22. MELISSARATOU, A.; FRIEDE, H. Dental arches and occlusion in bilateral cleft lip and palate patients after two different routines for palatal surgery. J. Orofac. Orthop., München, v. 63 , no. 4 , p. 300-314, July 2002

23. MOORREES, C. F. A. The dentition of the growing child: a longitudinal study of dental development between 3 and 18 years of age. Cambridge: Harvard University Press, 1959. cap. 6, p. 87-110.

24. NAVARRO, N. P. Estudo longitudinal das alterações dimensionais que ocorrem na distância intercanina, intermolar, dimensão vertical e comprimento do arco, em ambos os maxilares, no período da dentadura decídua e mista inicial, nos arcos tipo I e II de Baume, em crianças cubanas, na faixa etária de 5 a 7 anos e segundo o sexo. 1999. Dissertação (Mestrado)-Faculdade de Odontologia da USP, São Paulo, 1999

25. NYSTRÖM, M. The development of dental arches during the complete deciduous dentition period in a series of Finnish children studied with reference to chronologic and dental age. Proc. Finn. Dent. Soc., Helsinki, v. 75, no. 3, p. 27-34, 1979.

26. NYSTRÖM, M.; RANTA, R. Sizes of dental arches and interdental space in 3-year-old children with and without cleft lip/palate. Eur. J. Orthod., Oxford, v. 11, no. 1, p. 82-88, Feb. 1989.

27. NYSTRÖM, M.; RANTA, R. Effect of timing and method of cleft palate repair on dental arches at the age of three years. Cleft. Palate J., Pittsburgh, v. 27, no. 4, p. 349-352, Oct. 1990

28. SILVA FILHO, O. G. et al. Classificação das fissuras lábiopalatais: breve histórico, considerações clínicas e sugestão de modificação. Rev. Bras. Cir., Rio de Janeiro, v. 82, n. 2, p. 59-65, mar./abr. 1992

29. TOMANOVÁ, M.; MÜLLEROVÁ, Z. Growth of the dental arch in patients with complete unilateral cleft lip and palate after primary periosteoplasty. Acta Chir. Plast., Prague, v. 36, no. 4, p. 119-123, 1994

30. WADA, T.: MIZOKAWA, N.: MIYAZAKI, T.: ERGEN, G. Maxillary dental arch growth in different types of cleft. Cleft. Palate J., Pittsburgh, v. 21, no. 3, p. 180-192, July 1984.

\section{Endereço para correspondência} Juliana de Oliveira Romanelli Abi Faraj Av. Dr. Lineu Prestes, 2227 - Cidade Universitária CEP: 00.5508-900 - São Paulo / SP E-mail: jufaraj@usp.br 